\title{
Properties of the solar velocity field indicated by motions of coronal bright points
}

\author{
B. Vršnak ${ }^{1}$, R. Brajša ${ }^{1,2, \star}$, H. Wöhl ${ }^{2}$, V. Ruždjak ${ }^{1}$, F. Clette ${ }^{3}$, and J.-F. Hochedez ${ }^{3}$ \\ ${ }^{1}$ Hvar Observatory, Faculty of Geodesy, University of Zagreb, Kačićeva 26, 10000 Zagreb, Croatia \\ e-mail: [romanb; vruzdjak]@geodet.geof.hr \\ 2 Kiepenheuer-Institut für Sonnenphysik (KIS), Schöneckstr. 6, 79104 Freiburg, Germany \\ e-mail: rbrajsa@kis.uni-freiburg.de; hw@kis.uni-freiburg.de \\ 3 Observatoire Royal de Belgique (ORB), Av. Circulaire 3, 1180 Bruxelles, Belgium \\ e-mail: Frederic.Clette@oma.be; hochedez@oma.be
}

Received 22 January 2003 / Accepted 27 March 2003

\begin{abstract}
Full-disc solar images obtained with the Extreme Ultraviolet Imaging Telescope (EIT) on board the Solar and Heliospheric Observatory (SOHO) are used to analyse properties of the solar velocity field by tracing coronal bright points from June 4, 1998 to May 22, 1999. Rotation velocity residuals, meridional motions and their relationship are investigated. Zones of slow and fast rotation found in motions of coronal bright points are consistent with the pattern of torsional oscillations, indicating that the statistical velocity pattern of bright point motions reflects the large-scale plasma flows. A complex pattern of meridional motion is deduced: The equatorward flows are found to dominate at low $\left(B<10^{\circ}\right)$ and high $\left(B>40^{\circ}\right)$ latitudes, whereas at mid-latitudes $\left(B \approx 10^{\circ}-40^{\circ}\right)$ a poleward flow is inferred. The complete data set shows no significant correlation between rotation residuals and meridional motions. However, when a subsample of coronal bright points including only the "point-like structures" (predominantly young bright points) is considered, a statistically significant correlation is found. On average, faster tracers show equatorward motion and the slower ones show poleward motion. Such a segregation is reflected in a statistically significant covariance of the rotation residuals and meridional velocities in the order of $-1000 \mathrm{~m}^{2} \mathrm{~s}^{-2}$, revealing an equatorward transport of angular momentum. The negative value of the covariance is provided by the high velocity tail in the velocity distribution of point-like structures, representing less than $15 \%$ of the population. The latitude dependence of the covariance can be expressed as $Q=-62 B+200 \mathrm{~m}^{2} \mathrm{~s}^{-2}$ covering the range $B=0^{\circ}-60^{\circ}$.
\end{abstract}

Key words. Sun: rotation - Sun: UV radiation - Sun: corona

\section{Introduction}

Properties of the solar surface velocity field can be analysed by investigating the rotation velocity residuals and meridional motions of suitable tracers. An up-to-date summary of properties of the solar velocity field, including new helioseismic studies, and the implications for understanding the solar rotation and activity cycle was recently given by Stix (2002).

The rotation residual is a deviation of the rotational velocity from the mean differential rotation profile. The positive/negative residuals represent faster/slower rotation velocities than the average at a given latitude. Doppler velocity measurements revealed the so-called torsional oscillations (Howard \& LaBonte 1980; LaBonte \& Howard 1982) showing on both hemispheres two alternate zones of faster and slower rotation with respect to the mean differential rotation. During the solar activity cycle the velocity pattern migrates towards the equator

Send offprint requests to: $\mathrm{B}$. Vršnak,

e-mail: bvrsnak@geodet.geof.hr

^ Alexander von Humboldt Research Fellow at KIS. which is clearly exposed in the butterfly-diagram presentation. The average latitude of solar activity is located poleward of the faster zone, i.e., equatorward from the slower zone. The other pair of slow/fast zones is located at higher latitudes and is a precursor of the new solar cycle (Howard \& LaBonte 1983; Howard 1984).

The torsional oscillations were also found later on by tracing the photospheric magnetic field patterns using the crosscorrelation technique (Snodgrass 1991; Komm et al. 1993a) and by the time-distance helioseismology analysis of solar Dopplergrams (Beck et al. 2002). Furthermore, Tuominen et al. (1983) and Howard (1991b) have found some indications of torsional oscillations in the Greenwich Photoheliographic Results (GPR) and the Mt. Wilson sunspot data set, respectively. On the other hand, Brajša et al. (2002a) did not find signatures of torsional oscillations from measurements of stable recurrent sunspot groups from the GPR.

The meridional motions on the solar surface can be measured either utilizing the Doppler method (e.g., Lustig \& Wöhl 1990), or by various tracing methods (e.g., 
Lustig \& Wöhl 1991). The measurements are burdened by a large noise and data scatter especially for the Doppler method (Lustig \& Wöhl 1990). A review of sunspot measurements is given by Wöhl (2002). However, some general trends can be deduced and the first question is whether meridional motions are symmetric or antisymmetric with respect to the solar equator. Hanslmeier \& Lustig (1986) and Lustig \& Hanslmeier (1987) have found some indications of antisymmetric meridional motion of sunspots (equatorward at one hemisphere and poleward at the other). However, other studies based on sunspots indicated a predominant symmetric meridional motion (e.g., Balthasar et al. 1986; Lustig \& Wöhl 1994), whereas Howard \& Gilman (1986) found an interplay of both the symmetric and antisymmetric component.

In the symmetric case it is important to recognize if the motion is poleward or equatorward, and if it is directed towards or away from the average latitude of activity. Predominantly poleward motions in each solar hemisphere were found for photospheric magnetic features (Komm et al. 1993b; Latushko 1994; Snodgrass \& Dailey 1996) and in the subsurface layers using various helioseismic procedures (Beck et al. 2002; Haber et al. 2002). On the other hand, Howard (1991a) reported meridional motions of growing active regions towards the average latitude of activity. Similar behaviour was also found for growing sunspots (Howard 1991b; Lustig \& Wöhl 1994). However, the opposite motions, i.e., away from the average latitude of activity were reported for other data sets of growing sunspots (Wöhl \& Brajša 2001; Wöhl 2002). Decaying sunspots are moving away from the average latitude of activity (Howard 1991b; Wöhl \& Brajša 2001; Wöhl 2002). The pattern of meridional motions also shows a latitude drift during the solar cycle, as demonstrated by the butterfly diagram in, e.g., Snodgrass \& Dailey (1996) or Beck et al. (2002).

One of the most important questions is whether the rotation velocity residuals and meridional velocities are related. A particularly important parameter is the so-called covariance:

$Q=\left\langle\Delta v_{\text {rot }} v_{\text {mer }}\right\rangle$,

where the brackets represent averaging over the longitude (cf. Schröter 1985). The covariance $Q$ is supposed to expose the relative horizontal Reynolds stress associated with the Rossbytype eddies (see, e.g., Ward 1965) which might generate an equatorward transport of angular momentum (Ward 1965; Schröter 1985; Rüdiger 1989; Pulkkinen \& Tuominen 1998; Stix 2002). However, other possibilities in the case of, e.g., sunspots were also discussed by Gilman \& Howard (1984) and D'Silva \& Howard (1995). Although elusive in many studies, it seems that there exists a real transport towards the equator of the order of $10^{3} \mathrm{~m}^{2} \mathrm{~s}^{-2}$ (Schröter 1985; Stix 2002). A possible latitudinal dependence of the relative horizontal Reynolds stress was investigated and an increase with the latitude was found in some studies (Ward 1965; Gilman \& Howard 1984; D'Silva \& Howard 1995; Meunier et al. 1997; Pulkkinen \& Tuominen 1998).

In this paper we analyse properties of the solar velocity field, investigating the rotation velocity residuals and meridional motions that are determined by tracing coronal bright points in full-disc EIT (the Extreme-Ultraviolet Imaging Telescope on board of the Solar and Heliospheric Observatory, SOHO, Delabourdinière et al. 1995) images of the Sun. The relationship between the rotation residual and the meridional motion is also investigated. The measurements and data reduction are briefly described in Sect. 2 (for details see Brajša et al. 2001a, 2002b). The results are presented in Sect. 3 and discussed in Sect. 4. Conclusions are drawn in Sect. 5.

\section{Measurements and data reduction}

Coronal bright points are small isolated features of increased $\mathrm{X}$-ray and EUV intensity. To investigate their motion we use the EIT full-disc solar filtergrams taken in the line Fe XV at the wavelength of $28.4 \mathrm{~nm}$, recorded from June 4, 1998 to May 22, 1999. In the analysis we include only the periods which generally provide a 6-hour cadence (for details see Brajša et al. 2001a), leaving a set of 463 images, which were taken in June, November, and December 1998 and in March, April, and May 1999. For precise position measurements the improved solar disc coordinates (solar radius and disc centre) obtained with the refined solar limb detection method (Auchère et al. 1998) are used. We note also that Auchère et al. (2000) checked and found that the plate scale is constant across the field of view.

Measurements are performed applying two independent methods, an interactive and an automatic one. For details on the measurements, data reduction and the results on the solar differential rotation see the papers by Brajša et al. (2000, 2001a,b, 2002b) and by Wöhl et al. (2001). In Sects. 3.1-3.2 we present the results based on the interactive method since it provided a more coherent data set (more uniformly distributed over latitudes) and is thus more appropriate for the purposes of this study. These results are compared with the outcome of the automatic method in Sect. 3.3. We note that in the case of the interactive method we used the data set that includes tracers which are followed in up to 11 images (denoted as "data set 1" in Brajša et al. 2002b).

For each of the traced objects we determined the rotational velocity $\omega=\partial C M D / \partial t$ and the meridional motion $w_{y}=\partial B / \partial t$, where $C M D$ is the central meridian distance and $B$ the heliographic latitude. In the following the northern and southern hemisphere are treated together so we use a "symmetric representation", $w_{\mathrm{mer}}=-w_{y}$ for the southern hemisphere and $w_{\text {mer }}=w_{y}$ for the northern one: $w_{\text {mer }}>0$ always represents a poleward motion and $w_{\text {mer }}<0$ an equatorward motion, whereas $Q<0$ corresponds to the equatorward transport of angular momentum. All sidereal rotation velocities lower than $8 \mathrm{deg} \mathrm{day}^{-1}$ and higher than $18 \mathrm{deg} \mathrm{day}^{-1}$ were excluded. The number of data points obtained in this way is 1236 and 1710 for the interactive and the automatic method, respectively. In the interacive method the mean error of azimuthal velocity estimates (the slopes of individual $C M D(t)$ ) is $0.43 \mathrm{deg} \mathrm{day}^{-1}$ and in the case of the meridional velocities (the slopes of individual $\left.w_{y}(t)\right)$ is 0.25 deg day $^{-1}$. 


\section{Results}

\subsection{Large scale mean flows}

\subsubsection{Azimuthal flows}

The solar differential rotation $\omega(B)$ is determined in the form:

$\omega(B)=a+b \sin ^{2} B+c \sin ^{4} B$

where the northern and southern hemispheres are treated together, i.e., $|B|$ values are used in the fitting. The obtained sidereal values of the coefficients are: $a=14.477 \pm 0.046$, $b=-1.84 \pm 0.41$, and $c=-1.94 \pm 0.58$, expressed in deg day ${ }^{-1}$ (Fig. 1a).

For each measured object the rotation residual $\Delta \omega_{i}\left(B_{i}\right)=$ $\omega_{i}\left(B_{i}\right)-\omega\left(B_{i}\right)$ is determined, where $\omega\left(B_{i}\right)$ is defined by Eq. (2). The extreme values of rotation residuals are around \pm 6 deg day $^{-1}$ and the standard deviation is $\sigma_{\Delta \omega}=$ $0.94 \mathrm{deg} \mathrm{day}^{-1}$. The average absolute value of the residual velocity amounts to $\left\langle\left|\Delta v_{\text {rot }}\right|\right\rangle=0.66 \mathrm{deg} \mathrm{day}^{-1}$.

The obtained $\Delta \omega_{i}\left(B_{i}\right)$ data set is fitted by 4 th-6th degree polynomials which are shown in Fig. 1b. Note that the scale of the $y$-axis in Fig. 1b is stretched to expose the polynomials more clearly. The correlation coefficients for all fits amount to $C=0.045$, corresponding to a low statistical significance (see the confidence lines shown in Fig. 1b). However, all three polynomials show practically the same behaviour with amplitudes $\Delta \omega_{\text {poly }} \approx \pm 0.05 \mathrm{deg}_{\text {day }}{ }^{-1}$.

Figure $1 \mathrm{~b}$ indicates a slower-than-average rotation in the equatorial regions $\left(B<10^{\circ}\right)$, a belt of faster-than-average rotation between $B \approx 10^{\circ}$ and $B \approx 25^{\circ}$, and a slower rotation zone in the latitude range $25^{\circ}<B<50^{\circ}$. Beyond $B>50^{\circ}$ the number of data points becomes relatively small (see Fig. 1a and Table 1) so the fast rotation zone indicated by the polynomials therein is entirely ambiguous. Note that the latitude $B \approx 25^{\circ}$, separating the faster and slower rotation zone, corresponds closely to the mean latitude of sunspots in the considered period (marked by the dashed vertical line in Figs. 1-5).

The same qualitative behaviour for $B<50^{\circ}$ is found if the rotation residuals $\Delta \omega$ are presented by the azimuthal residual velocity $\Delta v_{\text {rot }}=\Delta \omega r_{B}=\Delta \omega r_{\odot} \cos B$, where $r_{\odot}$ is the solar radius and $\Delta \omega$ is expressed in $\mathrm{rad} \mathrm{s}^{-1}$. The mean azimuthal velocities $\overline{\Delta v}_{\text {rot }}(\bar{B})$ expressed in $\mathrm{m} \mathrm{s}^{-1}$ are shown in Fig. 1c for 5-degree and 10-degree bins of $B$ to check the influence of sampling. We also show the mean values calculated by grouping the data into six subgroups, each containing an equal number of $n=206$ data points (Table 2). Note the similarity of the results for the $n=206$ and the 10-deg sampling in the range $B<50^{\circ}$. The results are presented in the 4 th column of Table 1 , and 3 rd column of Table 2 . If the bin width is decreased to 5 degrees the scatter becomes considerably larger, but the general behaviour remains similar.

The graph in Fig. 1c shows that the difference between the maximum and minimum value of $\overline{\Delta v}_{\text {rot }}$ amounts to about $20 \mathrm{~m} \mathrm{~s}^{-1}$ in the case of the $n=206$ sampling. The standard deviations of the mean values are in the order of $\sigma_{v} \approx$ $\pm 100 \mathrm{~m} \mathrm{~s}^{-1}$ (see Table 2). Considering the data in the bins with the maximum and minimum $\overline{\Delta v}_{\text {rot }}$ as two samples, the t-test a)

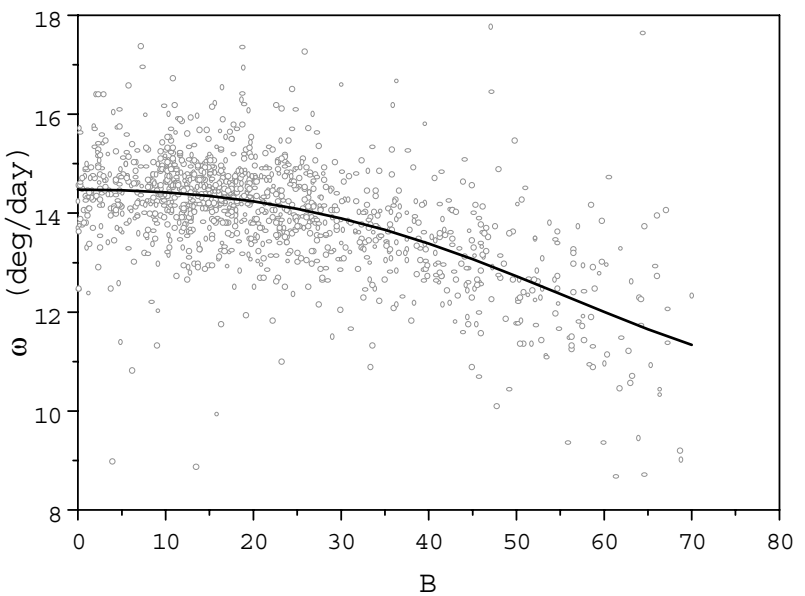

b)

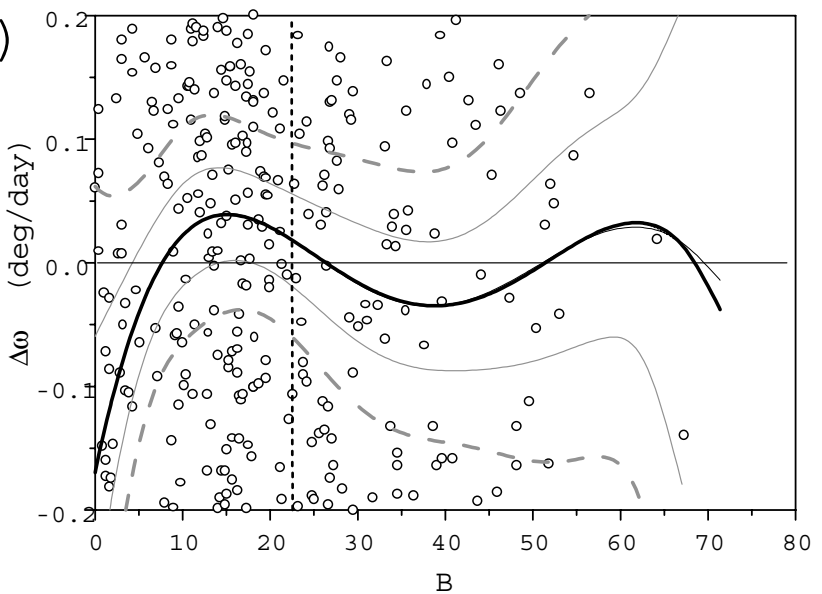

C)

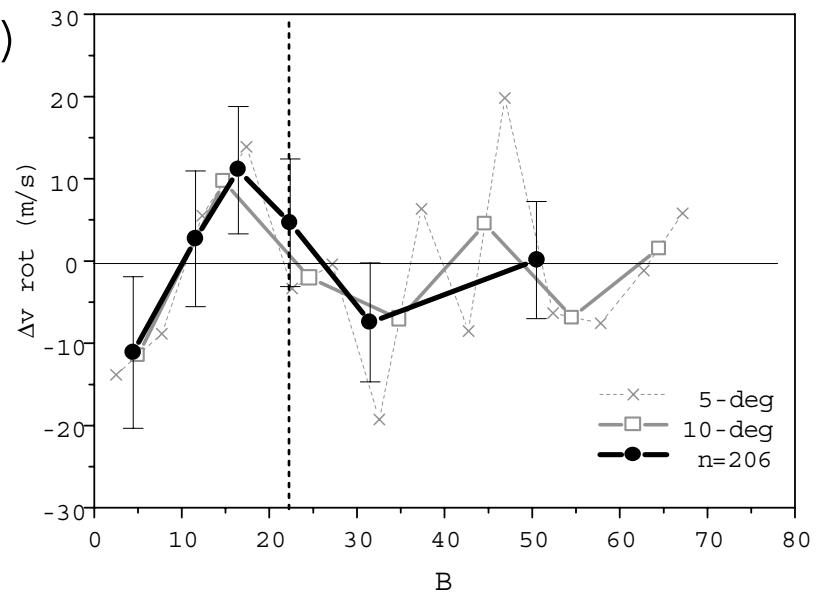

Fig. 1. a) The rotation $\omega(B)$ of EUV bright points. b) The angular velocity residuals $\Delta \omega(B)$. Note that the scale of the $y$-axis is stretched $77 \%$ of the data-points are out of scale. The 4th-6th degree polynomial least squares fits are drawn (the 4th degree polynomial is drawn bold; the 5th degree fit is hidden behind the 4th degree polynomial). The thick-dashed and the thin gray lines show the $95 \%$ and $65 \%$ confidence limits, respectively, for the 4th degree polynomial. c) Mean values $\overline{\Delta v}_{\text {rot }}(\bar{B})$ expressed in $\mathrm{m} \mathrm{s}^{-1}$, obtained averaging over 5-deg bins (crosses), 10-deg bins (squares), and bins embracing equal number of data points $(n=206$; bold). The vertical dashed line in $\mathbf{b}$ ) and $\mathbf{c}$ ) (and in the rest of the figures) represents the mean latitude of sunspots in the corresponding period. The error bars show the standard errors $\delta=\sigma / \sqrt{n}$ for the $n=206$ sampling, where $\sigma$ is the standard deviation. 
Table 1. Summary of 5-deg and 10-deg bin averages and standard deviations for the complete sample based on the interactive method.

\begin{tabular}{llllll}
\hline \hline$B$-bin & $n$ & $\langle B\rangle \mathrm{deg}$ & $\left\langle\Delta v_{\mathrm{rot}}\right\rangle \mathrm{m} \mathrm{s}^{-1}$ & $\left\langle v_{\mathrm{mer}}\right\rangle \mathrm{m} \mathrm{s}^{-1}$ & $\left\langle\Delta v_{\mathrm{rot}} v_{\mathrm{mer}}\right\rangle$ \\
\hline $0-5$ & 120 & $2.52 \pm 1.49$ & $-13.8 \pm 138.4$ & $-3.9 \pm 74.1$ & $+177 \pm 7930$ \\
$5-10$ & 114 & $7.73 \pm 1.48$ & $-8.8 \pm 126.4$ & $-9.5 \pm 71.9$ & $+858 \pm 8980$ \\
$10-15$ & 213 & $12.34 \pm 1.43$ & $+5.5 \pm 110.2$ & $+2.5 \pm 75.3$ & $+31 \pm 10023$ \\
$15-20$ & 209 & $17.39 \pm 1.50$ & $+13.9 \pm 115.6$ & $+9.1 \pm 65.9$ & $-468 \pm 8232$ \\
$20-25$ & 143 & $22.61 \pm 1.43$ & $-3.3 \pm 111.4$ & $+0.2 \pm 83.5$ & $-1144 \pm 12467$ \\
$25-30$ & 111 & $27.17 \pm 1.34$ & $-0.4 \pm 100.7$ & $+12.4 \pm 77.9$ & $+774 \pm 11561$ \\
$30-35$ & 69 & $32.55 \pm 1.43$ & $-19.2 \pm 109.1$ & $+6.2 \pm 80.0$ & $+2206 \pm 8693$ \\
$35-40$ & 63 & $37.38 \pm 1.58$ & $+6.4 \pm 104.4$ & $-10.3 \pm 64.3$ & $+315 \pm 9268$ \\
$40-45$ & 52 & $42.73 \pm 1.39$ & $-8.5 \pm 110.4$ & $+7.9 \pm 74.3$ & $-122 \pm 9140$ \\
$45-50$ & 44 & $46.87 \pm 1.38$ & $+19.8 \pm 130.7$ & $-10.7 \pm 89.1$ & $-1007 \pm 17209$ \\
$50-55$ & 40 & $52.37 \pm 1.63$ & $-6.3 \pm 77.1$ & $-25.4 \pm 104.7$ & $-1359 \pm 9927$ \\
$55-60$ & 27 & $57.82 \pm 1.26$ & $-7.6 \pm 91.2$ & $-19.1 \pm 91.5$ & $+1926 \pm 8684$ \\
$60-65$ & 19 & $62.72 \pm 1.58$ & $-1.2 \pm 135.9$ & $-21.75 \pm 89.6$ & $-686 \pm 6415$ \\
$65-70$ & 12 & $67.17 \pm 1.42$ & $+5.8 \pm 90.0$ & $+61.9 \pm 72.8$ & $+2916 \pm 10388$ \\
\hline $0-10$ & 234 & $5.06 \pm 3.00$ & $-11.4 \pm 132.4$ & $-6.6 \pm 73.0$ & $+531 \pm 8363$ \\
$10-20$ & 422 & $14.83 \pm 2.92$ & $+9.7 \pm 112.8$ & $+5.7 \pm 70.8$ & $-297 \pm 9293$ \\
$20-30$ & 254 & $24.60 \pm 2.66$ & $-2.0 \pm 106.6$ & $+5.6 \pm 81.2$ & $-557 \pm 11976$ \\
$30-40$ & 132 & $34.85 \pm 2.85$ & $-7.0 \pm 107.3$ & $-1.7 \pm 73.1$ & $+1593 \pm 9312$ \\
$40-50$ & 96 & $44.62 \pm 2.49$ & $+4.5 \pm 110.8$ & $-0.6 \pm 89.2$ & $-97 \pm 14160$ \\
$50-60$ & 67 & $54.56 \pm 3.08$ & $-6.8 \pm 82.4$ & $-22.9 \pm 99.0$ & $-289 \pm 9755$ \\
$60-70$ & 31 & $64.45 \pm 2.66$ & $+1.5 \pm 118.6$ & $+10.6 \pm 92.1$ & $-1809 \pm 9232$ \\
\hline
\end{tabular}

gives $P^{\prime}=1 \%$ for the probability that they represent underlying populations of equal mean values. This implies that the statistical significance (confidence level) of the difference between the two samples is $P=100-P^{\prime}=99 \%$. The deviation from the overall mean value $\overline{\Delta v}_{\text {rot }}=0$ (the amplitude), amounting to $10 \mathrm{~m} \mathrm{~s}^{-1}$, has the confidence level of $85 \%$. A similar behaviour is found for the 10-deg sampling in the range $B<50^{\circ}$. The variations exposed by the last three bins of the 10-deg sampling $\left(B>40^{\circ}\right)$ are statistically unreliable. This however does not imply that variations there do not exist (as indicated by the mean value of the last $n=206 \mathrm{bin}$, amounting to $\overline{\Delta v}_{\text {rot }} \approx 0$ ). Simply, our data set cannot provide an evidence for variations.

The variations exposed by the 5-deg sampling are generally of a low confidence level. The amplitude of $10 \mathrm{~m} \mathrm{~s}^{-1}$ in the case of a subsample containing 100 data points has a confidence level of only $68 \%$. Yet, in the $B<40^{\circ}$ range they show a similar variation as the $n=206$ and 10-deg subsamples.

\subsubsection{Meridional flows}

In Fig. 2 a the meridional velocity $w_{\text {mer }}$ expressed in deg day ${ }^{-1}$ is shown for each traced bright point. The object with $w_{\text {mer }}>$ 0 moves polewards, whereas $w_{\text {mer }}<0$ represents an equatorward motion. The mean value and the standard deviation for the complete data set amount to $\bar{w}_{\text {mer }}=0.005 \pm$ $0.55 \mathrm{deg} \mathrm{day}^{-1}$, implying that our data set does not show any

\footnotetext{
${ }^{1}$ Roughly speaking, in the $n=206$ sample the differences larger than $14 \mathrm{~m} \mathrm{~s}^{-1}$ have a significance larger than $95 \%$.
}

statistically significant net meridional flow. The extreme values are around $\pm 2 \mathrm{deg}_{\mathrm{day}^{-1}}$. The average absolute value of the meridional velocities amounts to $\left\langle\left|v_{\text {mer }}\right|\right\rangle=0.42$ deg day $^{-1}$, i.e. the $\left\langle\left|\Delta v_{\text {rot }}\right|\right\rangle$ is larger for about $60 \%$ than $\left\langle\left|v_{\text {mer }}\right|\right\rangle$.

In Fig. $2 b$ the same data are shown in a graph where the $y$-axis is stretched to expose more transparently the variation of the fitted 4th, 5th, and 6th degree polynomials. The correlation coefficients are $C_{4}=0.107, C_{5}=0.107$, and $C_{6}=0.117$, respectively, and the corresponding significances are in all three cases larger than $99 \%$ (the probability for $C=0$ is smaller than $1 \%$ ). Note that the three fits show very similar variations. The amplitude of the polynomials in the range $B<50^{\circ}$ is around $\Delta v_{\text {poly }}= \pm 0.07 \mathrm{deg}_{\text {day }}{ }^{-1}$.

In Fig. 2c the mean values of the meridional velocity $\bar{v}_{\text {mer }}(\bar{B})$, expressed in $\mathrm{m} \mathrm{s}^{-1}$, are shown. The same sampling as in the case of the rotation residuals is applied. Standard deviations of the mean values range between 60 and $100 \mathrm{~m} \mathrm{~s}^{-1}$ (see Table 1). The mean value and standard deviation of the meridional velocity for the complete data set amount to $\bar{v}_{\text {mer }}=$ $0.65 \pm 78 \mathrm{~m} \mathrm{~s}^{-1}$. Such a deviation from $\bar{v}_{\text {mer }}=0$ is statistically insignificant.

The mean values $\bar{v}_{\text {mer }}$ in the $B<50^{\circ}$ range reflect the behaviour of the polynomial fits shown in Fig. 2b: The motion of bright points for $B<10^{\circ}$ is on the average directed equatorwards, and in the range $10^{\circ}<B<40^{\circ}$ it is showing a poleward trend. Note that at the mean latitude of the sunspots the bright points show the poleward flow. On the other hand, in the equatorial region $\left(B<10^{\circ}\right)$, corresponding to a slower 
a)

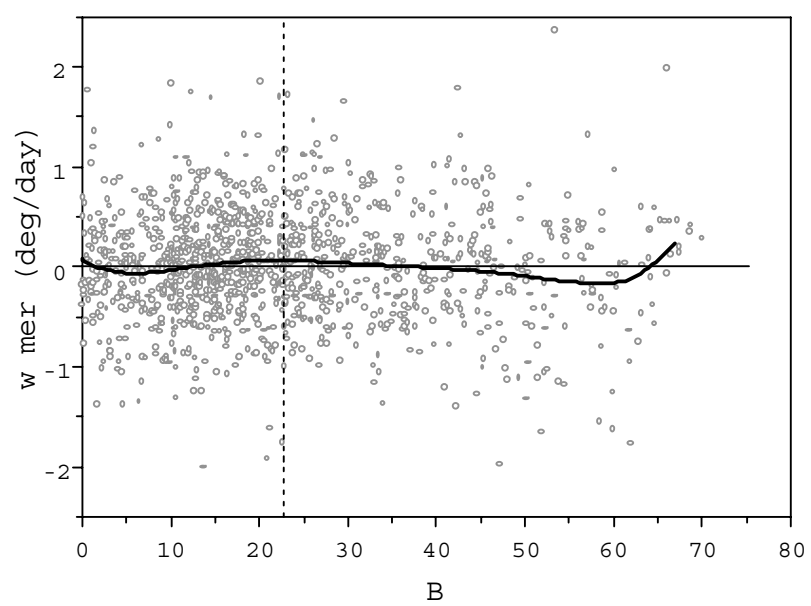

b)

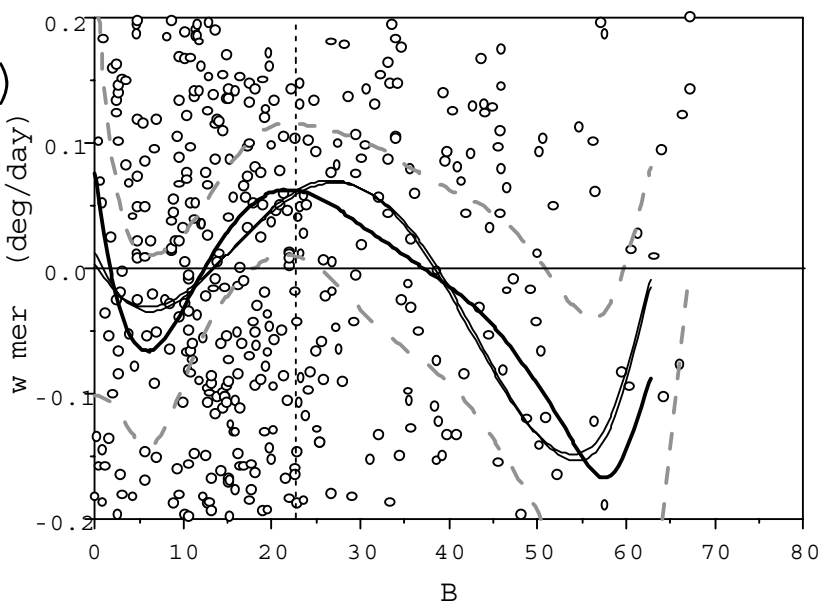

C)

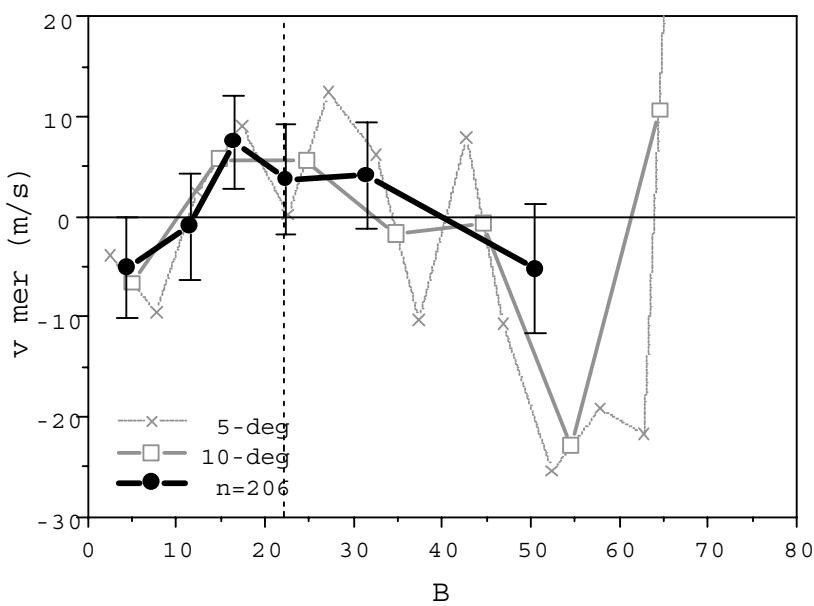

Fig. 2. a) The meridional motion $w_{\text {mer }}(B)$ of EUV bright points - full scale. b) The $w_{\text {mer }}(B)$ graph with the scale of the $y$-axis stretched $67 \%$ of the data-points are out of scale. The 4 th-6th degree polynomial least squares fits are shown (the 6th degree polynomial is drawn bold; the 4th and 5th polynomials are two closely spaced curves). The boldgray dashed line shows the $95 \%$ confidence limit for the 6 th degree polynomial. c) Bin-averaged meridional velocities $\bar{v}_{\text {mer }}(\bar{B})$ expressed in $\mathrm{m} \mathrm{s}^{-1}$, for 5-deg bins (crosses), 10-deg bins (squares), and bins embracing equal number of data points $(n=206$; bold). The error bars show the standard errors $\delta=\sigma / \sqrt{n}$ for the $n=206$ sampling, where $\sigma$ is the standard deviation. a)

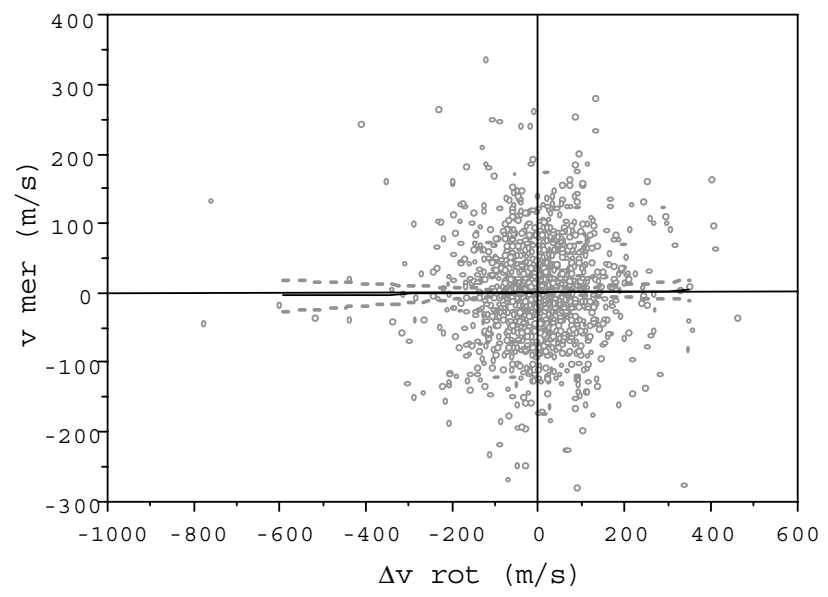

b)

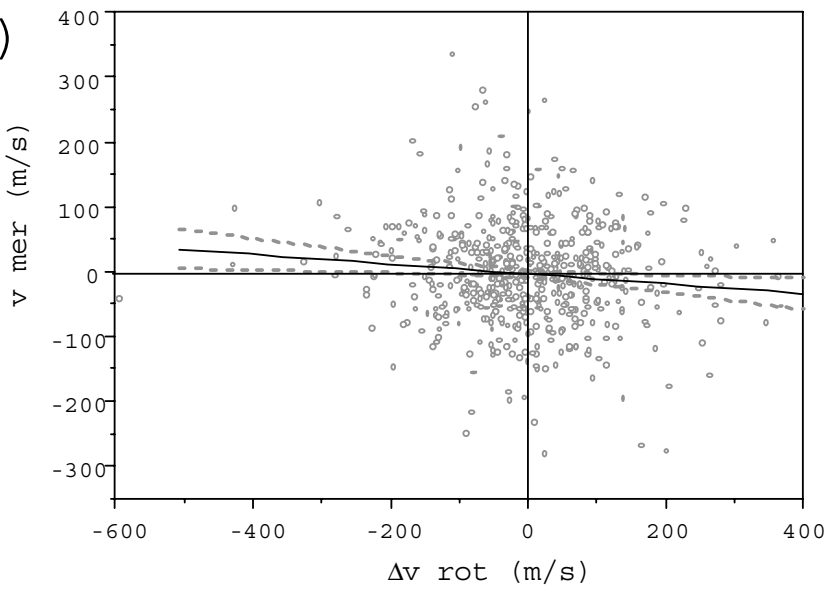

Fig. 3. The distribution in the $v_{\text {mer }}\left(\Delta v_{\text {rot }}\right)$ space. The linear least squares fit and the 95\% confidence limits are shown by black-full and gray-dashed lines, respectively. a) The complete sample; b) The PLS-sample (point-like structures). Note the different scales of the $x$-axes.

rotation zone, bright points show statistically a convergence of the flow towards the equator $\left(\bar{v}_{\text {mer }}<0\right)$. The data indicate again the equatorward motion in the range $60^{\circ}>B>40^{\circ}$ (see also the $95 \%$ confidence lines in Fig. 2b).

\subsection{Eddy flows}

In Brajša et al. (2002b) differences between the rotational characteristics of three morphologically different subtypes of coronal bright points, the "Point-Like Structures" (PLS), the "Small Loops" (SL), and the "Small Active Regions" (SAR), were investigated. It was demonstrated therein that the three types show a significantly different rotational pattern: SARs show a faster and "more rigid" rotation than PLSs. Furthermore, the three types have different lifetimes, SARs having longest duration (Brajša et al., in preparation), and they belong to different phases of a frequent evolutionary sequence of bright points, $\mathrm{PLS} \rightarrow \mathrm{SL} / \mathrm{SAR} \rightarrow \mathrm{PLS}$, that was recognized in the course of 
a)

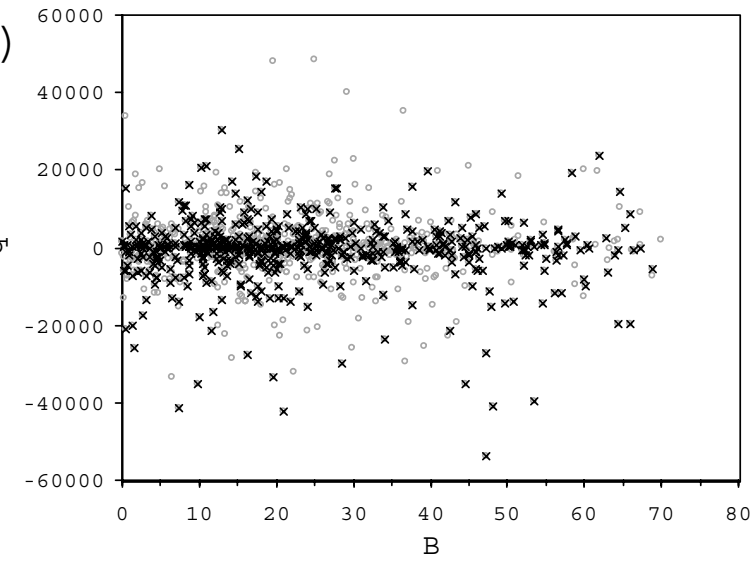

b)

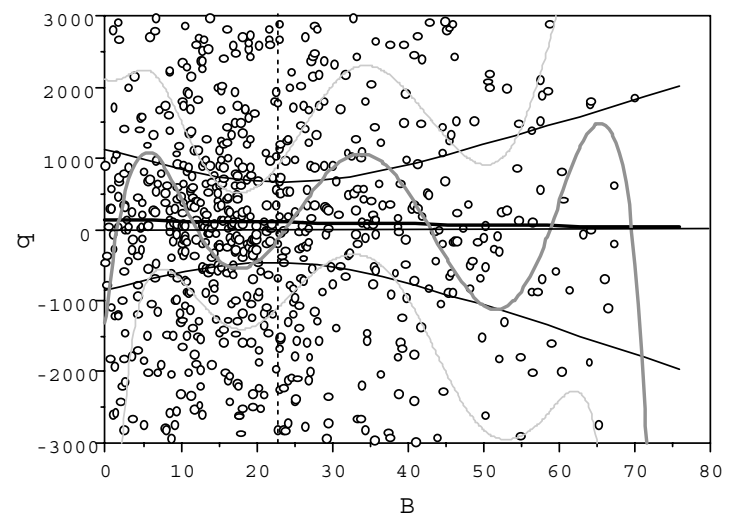

C)

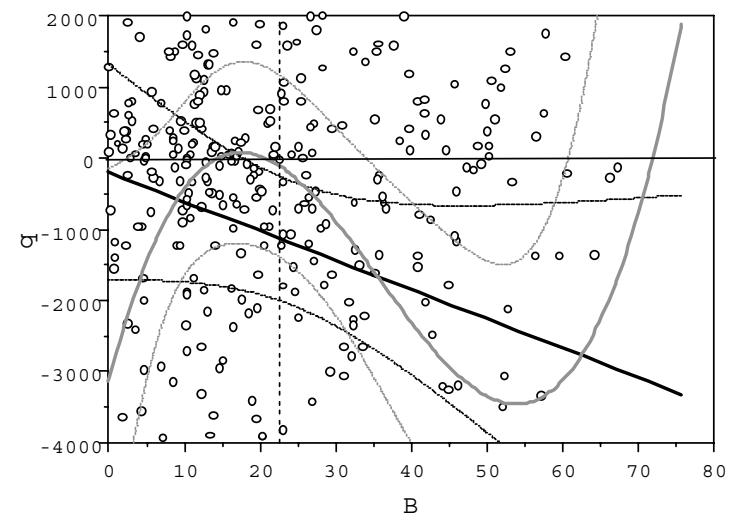

Fig. 4. The products $q=v_{\text {mer }} \Delta v_{\text {rot }}$, expressed in $\mathrm{m}^{2} \mathrm{~s}^{-2}$ and presented as a function of latitude. a) The complete sample, full scale - pointlike structures (PLS-sample) are enhanced by black crosses; b) The complete sample, stretched $y$-axis scale; c) The PLS-sample, stretched $y$-axis scale. The linear fits are drawn by thick-black lines whereas the 6th and 3rd degree polynomials shown in b) and c), respectively, are drawn by thick-gray lines. The $95 \%$ confidence limits are drawn by thin lines.

measurements by the interactive method ${ }^{2}$. Because of the possibility that objects of different lifetime/age can show different

\footnotetext{
2 A particular tracer was denoted as PLS, SL, or SAR according to the dominant (the longest duration) phase in its evolution during the tracing interval. For some illustrative examples of the evolution of bright points see, e.g., Fig. 2 in Brown et al. (2001), or Figs. 6 and 7 in Zhang et al. (2001).
}
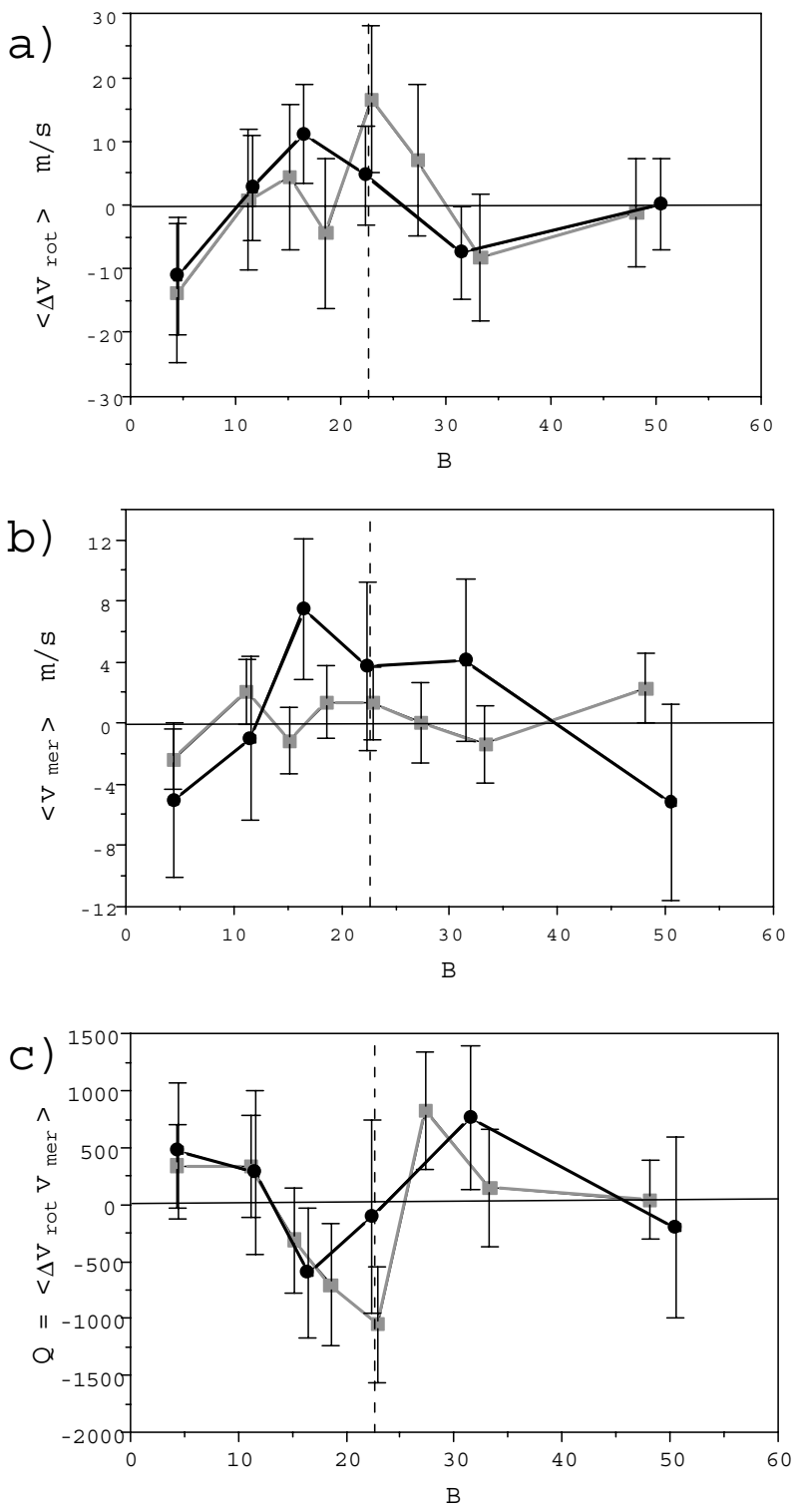

Fig. 5. Comparison of the bin-averaged values obtained from the interactive (black) and automatic (gray) method of measurements: a) Rotation velocity residual $\left\langle\Delta v_{\text {rot }}\right\rangle$; b) Meridional velocity $\left\langle v_{\text {mer }}\right\rangle$; c) The covariance $Q=\left\langle\Delta v_{\text {rot }} v_{\text {mer }}\right\rangle$. The error bars show the standard errors $\delta=\sigma / \sqrt{n}$, where $\sigma$ is the standard deviation.

Reynolds stress characteristics (Pulkkinen \& Tuominen 1998), we analyse the relationship between $\Delta v_{\text {rot }}$ and $v_{\text {mer }}$ not only using the complete sample, but also by considering the three morphological subtypes separately. Note that the PLS, SL, and SAR subsamples consist of 574, 200, and 462 tracers, respectively, so the results related to SLs are highly ambiguous due to the small number of tracers.

\subsubsection{Correlation of $v_{\text {mer }}$ and $\Delta v_{\text {rot }}$}

Figure 3 shows a distribution of the data in the $\left(v_{\text {mer }}, \Delta v_{\text {rot }}\right)$ space. When the complete data set is considered (Fig. 3a) the linear regression gives $v_{\text {mer }}=(0.008 \pm 0.019) \Delta v_{\text {rot }}+(0.07 \pm$ 2.2 ), which is statistically insignificant (see the $95 \%$ confidence 
Table 2. Comparison of the interactive ( $n=206$ bins) and automatic method ( $n=214$ bins).

\begin{tabular}{lllll}
\hline \hline bin & $\langle B\rangle$ deg & $\left\langle\Delta v_{\text {rot }}\right\rangle \mathrm{m} \mathrm{s}^{-1}$ & $\left\langle v_{\text {mer }}\right\rangle \mathrm{m} \mathrm{s}^{-1}$ & $\left\langle\Delta v_{\text {rot }} v_{\text {mer }}\right\rangle$ \\
\hline$n=206_{1}$ & $4.46 \pm 2.68$ & $-11.1 \pm 132.3$ & $-5.1 \pm 72.7$ & $+474 \pm 8626$ \\
$n=206_{2}$ & $11.58 \pm 1.36$ & $+2.7 \pm 118.4$ & $-1.0 \pm 76.8$ & $+280 \pm 10370$ \\
$n=206_{3}$ & $16.47 \pm 1.42$ & $+11.1 \pm 111.1$ & $+7.4 \pm 66.3$ & $-599 \pm 8189$ \\
$n=206_{4}$ & $22.37 \pm 2.02$ & $+4.6 \pm 111.4$ & $+3.7 \pm 79.4$ & $-104 \pm 12102$ \\
$n=206_{5}$ & $31.51 \pm 3.88$ & $-7.5 \pm 103.7$ & $+4.1 \pm 76.1$ & $+762 \pm 9143$ \\
$n=206_{6}$ & $50.49 \pm 8.07$ & $+0.1 \pm 102.2$ & $-5.2 \pm 92.2$ & $-204 \pm 11389$ \\
\hline \multicolumn{5}{c}{ automatic } \\
\hline$n=214_{1}$ & $4.38 \pm 2.50$ & $-13.9 \pm 160.2$ & $-2.4 \pm 28.6$ & $+341 \pm 5369$ \\
$n=214_{2}$ & $11.20 \pm 1.39$ & $+0.8 \pm 162.6$ & $+2.1 \pm 30.7$ & $+331 \pm 6583$ \\
$n=214_{3}$ & $15.16 \pm 1.01$ & $+4.3 \pm 166.0$ & $-1.1 \pm 32.4$ & $-312 \pm 6753$ \\
$n=214_{4}$ & $18.57 \pm 1.07$ & $-4.4 \pm 171.6$ & $+1.4 \pm 34.4$ & $-707 \pm 7829$ \\
$n=214_{5}$ & $22.91 \pm 1.29$ & $+16.6 \pm 168.0$ & $+1.3 \pm 34.9$ & $-1058 \pm 7399$ \\
$n=214_{6}$ & $27.34 \pm 1.31$ & $+7.0 \pm 174.6$ & $0.0 \pm 38.4$ & $+824 \pm 7551$ \\
$n=214_{7}$ & $33.28 \pm 2.20$ & $-8.3 \pm 145.2$ & $-1.4 \pm 37.2$ & $+146 \pm 7484$ \\
$n=214_{8}$ & $48.14 \pm 8.70$ & $-1.3 \pm 125.0$ & $+2.3 \pm 33.1$ & $+40 \pm 5070$ \\
\hline
\end{tabular}

limits in Fig. 3a). A similar outcome is found for the SL, SAR, and SL+SAR subsamples.

On the other hand, PLSs show a statistically significant correlation (see the $95 \%$ confidence limits in Fig. 3b). This outcome is not dependent on the procedure: If the rotation residuals are estimated relative to the differential rotation curve based only on the PLS-sample (the result shown in Fig. 3b) the linear regression gives $v_{\text {mer }}=(-0.076 \pm 0.030) \Delta v_{\text {rot }}-(3.9 \pm 3.3)$; If the differential rotation is based on the complete sample the fit gives $v_{\text {mer }}=(-0.076 \pm 0.030) \Delta v_{\text {rot }}-(4.6 \pm 3.3)$. Such a result indicates that there is a statistical "segregation" of PLSs - the faster ones move preferentially towards the equator, whereas the slower ones move polewards.

\subsubsection{The covariance $\left\langle\Delta v_{\text {rot }} v_{\text {mer }}\right\rangle$}

The $\left(\Delta v_{\text {rot }}, v_{\text {mer }}\right)_{i}$ data pairs are combined to get the values of the relative Reynolds stress products: $q_{i}=\Delta v_{i}^{\text {rot }} v_{i}^{\text {mer }}$. The results are presented in the $q(B)$ space in Fig. 4.

No significant dependence $q(B)$ is found for the complete sample. The linear least squares fit shown in Fig. $4 \mathrm{~b}$ gives $q=(-1.4 \pm 18.4) B+(133 \pm 509) \mathrm{m}^{2} \mathrm{~s}^{-2}$ with the correlation coefficient being only $C=0.002$, which corresponds to a $95 \%$ probability that there is no correlation $(C=0)$. Contrary to the case of $\Delta \omega(B)$ and $w_{\text {mer }}(B)$, where all fitted polynomials showed practically the same behaviour, here the fitted 4th, 5th, and 6th degree polynomials expose significant differences. The correlation coefficients are $C_{4}=0.01$, $C_{5}=0.04$, and $C_{6}=0.06$, respectively. The overall mean value $Q_{\mathrm{tot}}^{\text {all }}=\left\langle q_{i}\right\rangle=\left\langle\Delta v_{\mathrm{rot}} v_{\mathrm{mer}}\right\rangle$ for the complete sample amounts to $Q_{\text {tot }}^{\text {all }}=100 \pm 10000 \mathrm{~m}^{2} \mathrm{~s}^{-2}$. Such a deviation from $Q=0$ is insignificant.

The SL, SAR, and SL+SAR subsamples taken separately also do not show any statistically significant dependence $q(B)$, or deviation from $Q=0$. On the other hand, the PLSsample indicates a transport of angular momentum towards the equator, consistent with the $v_{\text {mer }}\left(\Delta v_{\text {rot }}\right)$ correlation result.
The linear least squares fit shown in Fig. $4 \mathrm{c}$ gives $q=(-41 \pm$ $26) B-(196 \pm 772) \mathrm{m}^{2} \mathrm{~s}^{-2}$ and the correlation coefficient of $C=0.07$ (Fig. 4c). The overall covariance (average over all latitudes) amounts to $Q_{\text {tot }}^{\text {PLS }}=-900 \pm 8200 \mathrm{~m}^{2} \mathrm{~s}^{-2}$. The fitted 3rd-6th degree polynomials show a very similar pattern and have the correlation coefficients $C_{3}=0.11, C_{4}=0.11$, $C_{5}=0.12$, and $C_{6}=0.14$, corresponding to significances ranging from $85 \%$ to $95 \%$.

The behaviour of the covariance $Q$ was also inspected utilizing the "standard" procedure (see, e.g., Ward 1965) by the latitudinal-bin averaging. For each bin the mean values of the rotation and meridional velocity, $\left\langle v_{\text {rot }}\right\rangle$ and $\left\langle v_{\text {mer }}\right\rangle$, were determined. These values were used to obtain for each tracer the value $\Delta v_{\text {rot }}^{*}=v_{\text {rot }}-\left\langle v_{\text {rot }}\right\rangle$ and $\Delta v_{\text {mer }}^{*}=v_{\text {mer }}-\left\langle v_{\text {mer }}\right\rangle$ which are then used to estimate the covariance $Q^{*}=\left\langle v_{\text {rot }}^{*} \Delta v_{\text {mer }}^{*}\right\rangle$. The overall mean value amounts to $Q_{\text {tot }}^{* \mathrm{PLS}}=-1250 \pm 10600 \mathrm{~m}^{2} \mathrm{~s}^{-2}$. The probability that the mean value is $\bar{Q}=0$ is $P^{\prime}<5 \%$.

The results for the 10-deg bins are shown in Table 3 (see also Fig. 6c). The linear least squares fit gives $Q^{*}=(-61 \pm$ $39) B+(167 \pm 1373) \mathrm{m}^{2} \mathrm{~s}^{-2}$. Since the bins contain a small number of data points we also calculated the value of $Q^{*}$ for $B<$ $30^{\circ}$ and $B>30^{\circ}$ and we obtain: $Q^{*}=-680 \pm 8310 \mathrm{~m}^{2} \mathrm{~s}^{-2}$ at $\bar{B}=14.25^{\circ}(n=399$ tracers $)$ and $Q^{*}=-2600 \pm 19000 \mathrm{~m}^{2} \mathrm{~s}^{-2}$ at $\bar{B}=45.49^{\circ}\left(n=175\right.$ tracers) giving $Q^{*}=-62 B+200$ (see also Fig. 6c).

\subsection{Comparison with the automatic method}

Proper motions of coronal EUV bright points were studied also by tracing their positions automatically (for the details of the automatic method see Brajša et al. 2001a, 2002b; Wöhl et al. 2001). This method provided a statistically different data set embracing 1710 objects. The latitudinal distribution in this set is less uniform than in the case of the interactive method - the majority of the bright points $(75 \%)$ is found in the active region zone $10^{\circ}<B<40^{\circ}$ (see the histograms in Brajša et al. 2003). Furthermore, we stress that the automatic method uses 

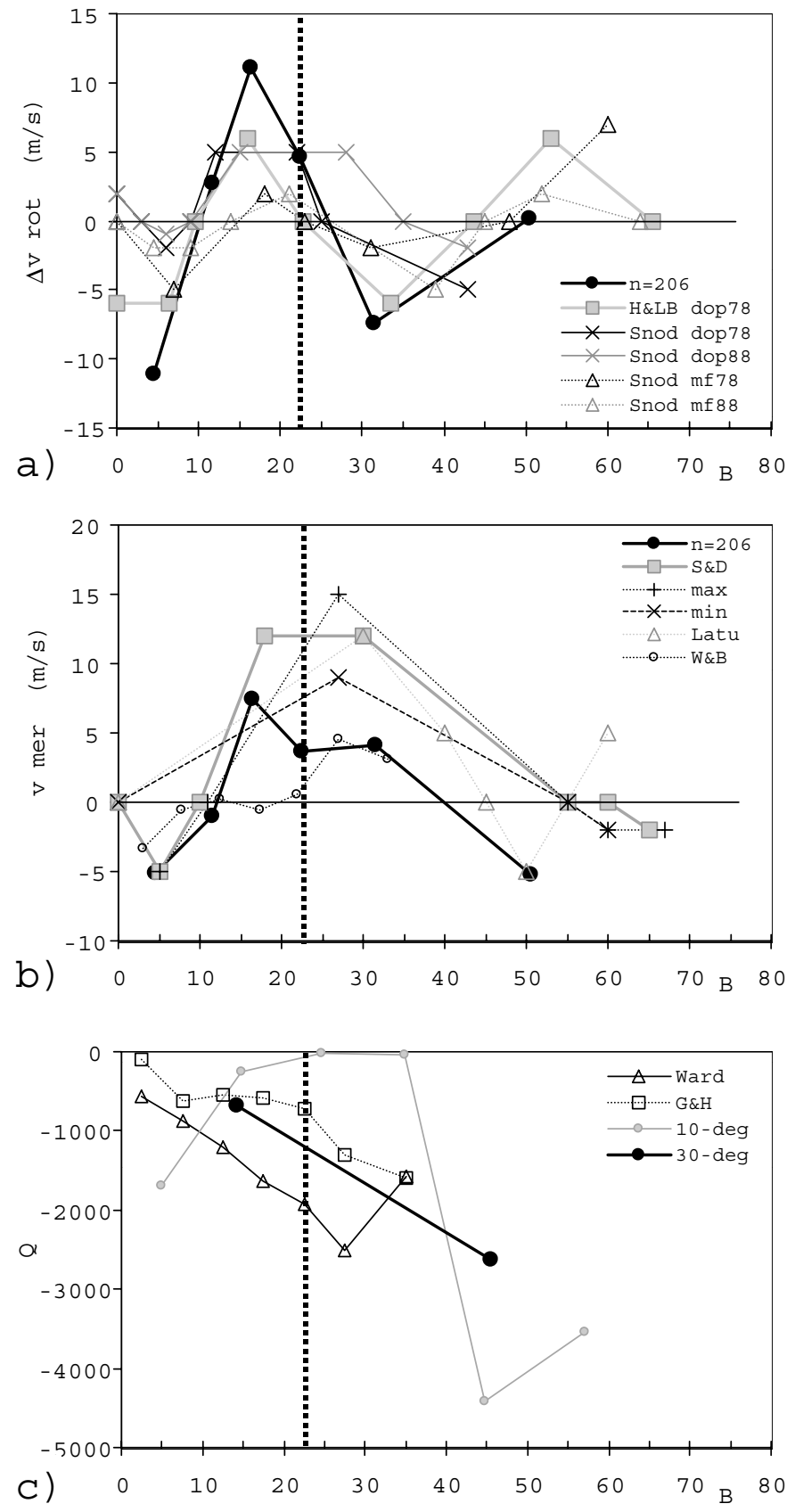

Fig. 6. a) Mean values ( $n=206$ sampling) of the rotation residual $\overline{\Delta v}_{\text {rot }}(\bar{B})$ compared with the results by Howard \& Labonte (1980) and Snodgrass (1991). b) Mean values of the meridional velocities $\bar{v}_{\text {mer }}(\bar{B})$ compared with results by Latushko (1994), Snodgrass \& Dailey (1996), and Wöhl \& Brajša (2001), denoted in the legend by "Latu", "S\&D", and "W\&B", respectively. The smoothed "S\&D" results are also shown separately for the solar cycle maximum ("max") and minimum ("min"). c) The covariance $Q=\left\langle\Delta v_{\mathrm{rot}} v_{\mathrm{mer}}\right\rangle$, expressed in $\mathrm{m}^{2} \mathrm{~s}^{-2}$. The PLS-sample is shown for 10-deg and 30-deg bins compared with the sunspot results by Ward (1965) and Gilman \& Howard (1984) which are denoted in the legend as "Ward" and "G\&H" and drawn by triangles and squares, respectively.

sequences of only three successive images, so the accuracy of velocity evaluation for a given tracer is substantially lower than in the interactive method.
Table 3. Covariances and the standard deviations for the subset containing only point-like structures (PLS-sample).

\begin{tabular}{llll}
\hline \hline$B$-bin & $n$ & $\langle B\rangle$ deg & $\left\langle\Delta v_{\text {rot }} v_{\text {mer }}\right\rangle_{\mathrm{PLS}} \mathrm{m}^{2} \mathrm{~s}^{-2}$ \\
\hline $0-10$ & 127 & $4.9 \pm 3.1$ & $-1689 \pm 7413$ \\
$10-20$ & 164 & $14.7 \pm 2.9$ & $-258 \pm 9133$ \\
$20-30$ & 108 & $24.6 \pm 2.8$ & $-18 \pm 6587$ \\
$30-40$ & 60 & $34.9 \pm 2.7$ & $-40 \pm 7489$ \\
$40-50$ & 56 & $44.7 \pm 2.7$ & $-4407 \pm 16615$ \\
$B>50$ & 59 & $57.0 \pm 5.2$ & $-3542 \pm 18544$ \\
\hline$B<30$ & 399 & $14.3 \pm 8.1$ & $-683 \pm 8306$ \\
$B>30$ & 175 & $45.5 \pm 9.9$ & $-2618 \pm 19003$ \\
\hline
\end{tabular}

In Fig. 5 and Table 2 we present a comparison of the bin-averaged values of the rotation velocity residuals $\overline{\Delta v}_{\text {rot }}(\bar{B})$, meridional velocity $\bar{v}_{\text {mer }}(\bar{B})$, and the covariance $Q=\left\langle\Delta v_{\text {rot }} v_{\text {mer }}\right\rangle$. In particular we compare the $n=206$ sampling in the interactive data set with the outcome for an analogous sampling in the automatic data set (eight subgroups each containing $n=214$ objects). Note that the automatic method does not provide an information about the morphological characteristics of bright points, so we can compare only the full data sets.

The results concerning the rotation residuals and covariances show a relatively good correspondence (Fig. 5a, c). In meridional velocities there are however important differences (Fig. 5b) - the automatic method does not show any statistically significant net meridional velocity or its variation. This could be attributed to the too restrictive criteria used for the identification of bright points in two successive images (Brajša et al. 2002b): Inspecting Table 2 one finds that the standard deviations of rotation residuals are considerably larger in the automatic method, whereas in the case of meridional velocities standard deviations are 2-3 times larger in the interactive method. This implies that a significant fraction of events with large meridional velocities is a priori rejected in the automatic method, cutting-out parts of the $v_{\text {mer }}$ bin-distributions, and decreasing the fluctuations of bin-averages $\bar{v}_{\text {mer }}(B)$.

On the other hand, due to a larger number of events in a given bin and an apparently larger amplitude (see Fig. 5a), the significance of the variations of mean rotational velocity residuals appear to be larger in the automatic method than in the case of the interactive method. For example, the deviation of $\Delta v_{\text {rot }}=+15 \mathrm{~m} \mathrm{~s}^{-1}$ at $B=22.9^{\circ}$ from $\overline{\Delta v}_{\text {rot }}=0$ corresponds to the confidence level of $97 \%$, being considerably larger than the value of $85 \%$ found for the interactive method (see Sect. 3.1).

Finally, let us clarify an apparent contradiction between Fig. 5c and Figs. 5a and b: One could (erroneously) expect that the sign of $Q$ at a particular $B$ is the same as the sign of the product of $\left\langle\Delta v_{\text {rot }}\right\rangle$ and $\left\langle v_{\text {mer }}\right\rangle$ values at that $B$, which is obviously not the pattern appearing in Fig. 5. However, it should be kept in mind that Figs. 5a and 5b describe the large scale mean flows, whereas Fig. 5c represents $Q$, which is based on the sum of individual products $\left(\Delta v_{\text {rot }} \times v_{\text {mer }}\right)_{i}$. This means that the sign of $Q$ is not necessarily the same as that of the product $\left\langle\Delta v_{\text {rot }}\right\rangle \times\left\langle v_{\text {mer }}\right\rangle$. This can be illustrated by considering, e.g., the simplest situation where $\Delta v_{\text {rot }}$ and $v_{\text {mer }}$ form normal distributions centered 
at $\left\langle\Delta v_{\text {rot }}\right\rangle=+c$ and $\left\langle v_{\text {mer }}\right\rangle=+c$. At the same time, let us take that all objects with $\Delta v_{\text {rot }}>0$ have $v_{\text {mer }}<0$, and vice versa. One finds that $Q<0$, whereas $\left\langle\Delta v_{\text {rot }}\right\rangle\left\langle v_{\text {mer }}\right\rangle>0$. This becomes especially prominent when $\left\langle\Delta v_{\text {rot }}\right\rangle^{2} \approx\left\langle v_{\text {mer }}\right\rangle^{2} \approx c^{2} \ll|Q|$, as in our case.

\section{Discussion: A comparison with previous studies}

\subsection{The rotation residual}

In Fig. 6a the bin-averaged values $\overline{\Delta v}_{\text {rot }}(\bar{B})$, obtained for the $n=206$ sampling of the interactive method are compared with the results of several previous studies.

Zones of fast and slow rotation were deduced by Howard \& Labonte (1980) by analysing the Doppler velocity measurements in the period 1968-1979. From their butterfly diagram showing the torsional oscillations we cut-out the year 1978 representing the phase of the solar cycle that corresponds to the phase of the time interval covered by our measurements. For this period we overlapped the north and south hemisphere results and estimated approximately the $\Delta v_{\text {rot }}(B)$ dependence by finding the (mean) latitudes at which $\Delta v_{\text {rot }}(B)=0, \Delta v_{\text {rot }}(B)=$ $\max$, and $\Delta v_{\text {rot }}(B)=\min$. The outcome is shown in Fig. 5a by gray squares connected by the gray line and denoted in the legend as "H\&LB-dop78".

The same procedure is applied to the results reported by Snodgrass (1991) which are obtained by the Doppler measurements and by "tracing" the magnetic field patterns for the period 1968-1990. In Fig. 5a we show the outcome of both methods (denoted as "Snod-dop" and "Snod-mf", respectively) for the corresponding phases of the solar cycles 21 and 22 (denoted as "78" and " 88 " to identify the year).

The outcome of all data sets is qualitatively, and in a great deal quantitatively, similar. Qualitatively, our results also partly agree with the rotation residual velocities of sunspots (Howard 1991b) and with zonal velocities in the subphotospheric levels (Haber et al. 2001, 2002).

The behaviour of the bin-averaged rotation velocity residuals exposed in Fig. 6a can be summarized as follows:

$$
\begin{aligned}
& -\left\langle\Delta v_{\text {rot }}\right\rangle<0 \text { at } B<10^{\circ} \\
& -\left\langle\Delta v_{\text {rot }}\right\rangle>0 \text { at } 10^{\circ}<B<20^{\circ} \\
& -\left\langle\Delta v_{\text {rot }}\right\rangle \approx 0 \text { at } B \approx \bar{B}_{\mathrm{s}} \text { (mean sunspot latitude) } \\
& -\left\langle\Delta v_{\text {rot }}\right\rangle<0 \text { at } 30^{\circ}<B<40^{\circ} \\
& -\left\langle\Delta v_{\text {rot }}\right\rangle \approx 0 \text { at } 40^{\circ}<B<50^{\circ} \\
& -\left\langle\Delta v_{\text {rot }}\right\rangle>0 \text { at } 50^{\circ}<B<60^{\circ} .
\end{aligned}
$$

The amplitude of the deviation from $\left\langle\Delta v_{\text {rot }}\right\rangle=0$ has the value between 5 and $10 \mathrm{~m} \mathrm{~s}^{-1}$.

\subsection{Meridional flows}

In Fig. $6 \mathrm{~b}$ the bin-averaged values $\bar{v}_{\text {mer }}(\bar{B})$ for the $n=$ 206 sampling are compared with the results reported by Latushko (1994), Snodgrass \& Dailey $(1996)^{3}$, and

${ }^{3}$ We have tried to use the butterfly-diagram presentation given in Fig. 2 in Snodgrass \& Dailey (1996) and to perform an analogous
Wöhl \& Brajša (2001). These results are denoted in the legend of Fig. 5b as "Latu", "S\&D", and "W\&B". The smoothed results of Snodgrass \& Dailey (1996) for the northern hemisphere are also shown in the form as presented by Snodgrass \& Smith (2000) separately for the solar maximum and minimum (denoted in the legend as " $m a x "$ and "min").

The overall behaviour of all results presented in Fig. $6 \mathrm{~b}$ is similar: All of the results show $v_{\text {mer }}>0$ between $B=10^{\circ}$ and $B=40^{\circ}$, with the maximum speed of $5-10 \mathrm{~m} \mathrm{~s}^{-1}$ for $B \approx 15^{\circ}-25^{\circ}$. Note that this range of latitudes corresponds roughly to the central latitude of sunspots. Our results indicate that for $B<10^{\circ}$ the EUV bright points show converging motion towards the equator, confirming the results by Snodgrass $\&$ Dailey (1996). If the motion of bright points reflects the surface plasma motion, this implies (equation of continuity) that there should be a downward sink of the material. Analogously, at $B \approx 10^{\circ}$ there should be an outflow of the material from below. Finally, our results indicate also a possible equatorward motion at high latitudes like in Snodgrass \& Dailey (1996), indicating a sink of material at mid-latitudes and an outflow at the poles.

Equatorward motions at low latitudes and poleward motions at medium latitudes, similar to that found herein, were reported also in a number of studies based on tracing sunspots (Balthasar et al. 1986; Howard 1991b; Lustig \& Wöhl 1994; Pulkkinen \& Tuominen 1998; Brajša \& Wöhl 2000; Wöhl \& Brajša 2001; Wöhl 2002).

An overall poleward motion was inferred by Komm et al. (1993b) for small photospheric magnetic features and by Haber et al. (2001, 2002) and Beck et al. (2002) for the subphotospheric layers. We note that, although small and statistically not significant, the mean value of meridional velocity found herein also shows such a poleward trend.

The behaviour of the meridional velocity exposed by Fig. $6 \mathrm{~b}$ can be summarized as follows:

$$
\begin{aligned}
& -\left\langle v_{\text {mer }}\right\rangle<0 \text { at } B<10^{\circ} \\
& -\left\langle v_{\text {mer }}\right\rangle>0 \text { at } 10^{\circ}<B<40^{\circ} \\
& -\left\langle v_{\text {mer }}\right\rangle \approx 0 \text { at } 40^{\circ}<B<50^{\circ} \\
& -\left\langle v_{\text {mer }}\right\rangle<0 \text { at } B>\left(50^{\circ}\right) .
\end{aligned}
$$

The amplitude of the variations lies between 5 and $10 \mathrm{~m} \mathrm{~s}^{-1}$.

\subsection{Relationship between $\Delta v_{\text {rot }}$ and $v_{\text {mer }}$}

In Fig. $6 \mathrm{c}$ the covariances for $10^{\circ}$ bins, $B<30^{\circ}$, and $B>30^{\circ}$ are shown, compared with the results by Ward (1965) and Gilman \& Howard (1984). The data taken from Ward (1965) are based on sunspot group tracing, whereas from Gilman \& Howard (1984) we took the individual sunspot measurements (for the discussion see Gilman \& Howard 1984). Note that coronal bright points provide an extension of covariance analysis up to polar regions.

procedure as in the case of the rotation velocity residuals (Sect. 4.1.1.). However, the velocity pattern is much more complex, and only a qualitative comparison is possible. For this reason we compare only the time-integrated results shown in Fig. 1 therein. 


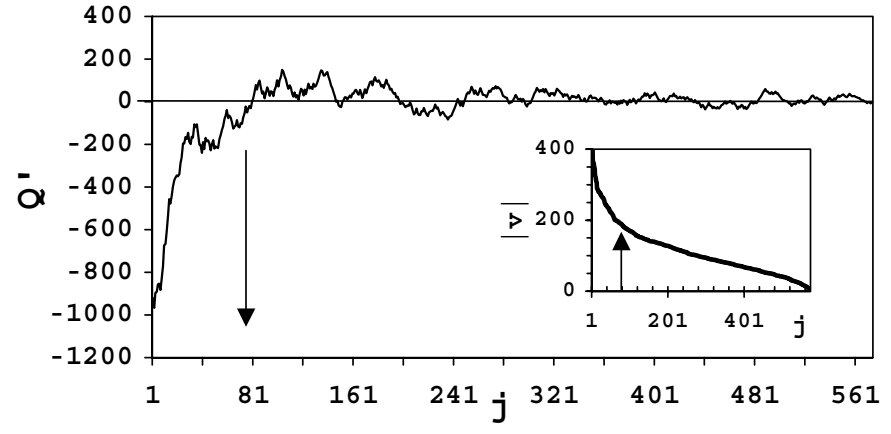

Fig. 7. The change of the covariance $Q_{|v|}^{\prime}(j)$ by successive elimination of tracers from high to low velocities. On the $x$-axis the ordinal number $j$ of the tracers arranged by descending velocities is given (larger $j$ means smaller velocity $|v|)$. The $y$-axis represents the covariance $Q^{\prime}(j)$ calculated using only the events with ordinal numbers $\geq j$. The $|v|(j)$ arrangement is shown in the inset - note that it also represents an inverted cumulative distribution of $|v|$. The downward directed arrow indicates $j \approx 80$ beyond which $Q \approx 0 \approx$ const. The arrow in the inset indicates $|v| \approx 200 \mathrm{~m} \mathrm{~s}^{-1}$ at $j=80$.

Inspecting Fig. 6c one finds that our results are sited roughly in between the two compared data sets, matching slightly better with the individual sunspot results. Note that the amount of the angular momentum transport found by tracing sunspot groups is probably overestimated (see, e.g., Gilman \& Howard 1984, or Pulkkinen \& Tuominen 1998 and references therein).

An intriguing outcome of the presented analysis is that not all bright point types show $Q<0$, but rather only the subsample based on PLSs. The PLS sample involves generally bright points of short lifetimes, with only a brief or no SAR/SL episode (Sect. 3.2). On the other hand, SAR and SL subsamples contain longer lasting tracers with a prominent SAR/SL phase, following the initial PLS stage. Note furthermore, that the duration of the tracing interval was limited (Sect. 2). Consequently, the long-lifetime tracers that were classified as PLSs due to the prevalence of the "late-PLS-phase" in the PLS $\rightarrow$ SL/SAR $\rightarrow$ PLS sequence (see Sect. 3.2) are at least partly excluded. All of this indicates that the PLS subsample represents a younger population than the SAR and SL subsamples. This is also reflected in their average lifetimes: the tracers classified as PLSs have on average shorter lifetimes than those classified as SARs (Brajša et al., in preparation).

It is also important to note that $Q<0$ is primarily provided by the PLSs with large values of $|q|=\left|\Delta v_{\text {rot }} v_{\text {merl }}\right|_{i}$, i.e., by the tail in the distribution of $q$. The $q<0$ tail is considerably more prominent than the $q>0$ tail (compare the distribution of PLS data-points in Fig. 4a below and above $q=0$ ). All of PLSs with a large $|q|$ also have large speeds $v \equiv|v|=\left(\Delta v_{\text {rot }}^{2}+v_{\text {mer }}^{2}\right)^{1 / 2}$. Eliminating successively PLSs with a large value of $|q|$, or equivalently, large $|v|$, the value of $Q$ is gradually diminishing, i.e., $Q \rightarrow 0$.

To demonstrate such a behaviour the $q(|v|)$ data set is arranged according to the value of $|v|$, so that successively lower velocities have respectively higher ordinal numbers, from $j=1$ (highest velocity) to $j=574$ (lowest velocity). Then, for each $j$ the covariance $Q_{|v|}^{\prime}(j)=\langle q\rangle_{j}$ was calculated using only the data with ordinal numbers $\geq j$. The outcome is shown in Fig. 7, where it can be seen that $Q \approx 0 \approx$ const. beyond $j \approx 80$, corresponding to $|v|<200 \mathrm{~m} \mathrm{~s}^{-1}$ (indicated in the inset in Fig. 7). Similarly, an analogous procedure was performed by arranging the $q(|q|)$ data, i.e., attributing successively higher ordinal numbers $k$ to successively lower values of $|q|$, and calculating $Q_{|q|}^{\prime}(k)$. From the graph $Q_{|q|}^{\prime}(k)$, one finds $Q \approx 0 \approx$ const. beyond $k \approx 70$ corresponding to $|q|<10000 \mathrm{~m}^{-2} \mathrm{~s}^{-2}$. This means that, in fact, only a subset of PLSs with high speeds, embracing $\approx 12-14 \%$ of all PLSs $(70 / 574$ and $80 / 574$, for the $Q_{|v|}^{\prime}(j)$ and $Q_{|q|}^{\prime}(k)$, respectively), gives rise to $Q<0$, whereas the rest, representing the majority of PLSs shows $Q=0$.

Bearing this in mind one can put forward the hypothesis that only newly generated elements of turbulence are contributing to the Reynolds stress: The transfer of the angular momentum to the large scale mean flow, from the small-scale short-living turbulence that is revealed by the motions of bright points, should proceed quite fast (say, a fraction of a day), since the majority of bright points falls into the category characterized by $Q=0$.

Another interesting point to discuss is the $Q(B)$ dependence. Bright points enable an observational estimate of the Reynolds stress at considerably larger latitudes than sunspots do. In this respect it is worth comparing our results with some theoretical considerations. According to the model by Canuto et al. (1994), the covariance $\left\langle\Delta v_{\text {rot }} v_{\text {mer }}\right\rangle$ should have a maximum value at the latitude $B \approx 30^{\circ}$. The PLS sample shows an extreme in the $Q(B)$ relationship at $B \approx 50^{\circ}$ (Fig. 4c), i.e., at considerably higher latitudes than predicted by Canuto et al. (1994). Yet, we emphasize that in this latitudinal range the results based on the PLS sample are ambiguous (see the 10-deg bin sampling in Fig. 6c) due to a relatively small number of data-points. So unfortunately, no definite conclusion can be drawn in this respect before a more extensive data set is prepared. Nevertheless, the "two-bin sampling" shown in Fig. 6c by the bold line shows that the covariance is growing up to $B \approx 45^{\circ}$, i.e. beyond the maximum predicted by Canuto et al. (1994). On the other hand, note that the "operational form" of the model by Canuto et al. (1994), denoted as M5, utilizes several approximations (e.g., the meridional flow and the radial gradient of the rotational velocity are neglected), and it is not clear how much these approximations affect the predicted location of the extreme in the $Q(B)$ dependence.

\section{Summary and conclusions}

The basic results obtained in this paper can be summarized as follows:

- Rotation residuals of coronal bright points show a pattern consistent with the torsional oscillations, indicating that the obtained velocity pattern reflects the large scale plasma flows.

- Equatorward motions are found at low and high latitudes, whereas in the active region belt poleward motions dominate. These results are qualitatively consistent with other studies performed with different methods. The best agreement is found with the results by 
Snodgrass \& Dailey (1996) who followed the magnetic features in the solar photosphere.

- The complete sample does not show a statistically significant net transport of angular momentum.

- Yet, a covariance consistent with the equatorward transport of angular momentum $(Q<0)$ is found in the subset that contains only point-like structures, generally representing a younger population of bright points.

- Tracing of coronal bright points provides an extension of the Reynolds stress analysis to high latitudes which could be an important tool to investigate the dependence of the Reynolds stress on the latitude.

The abovementioned properties of the solar velocity field are obtained applying the interactive method of tracing coronal bright points. This method turned out to be reliable enough to detect large scale flows of an amplitude even smaller than $10 \mathrm{~m} \mathrm{~s}^{-1}$. Measurements are comparatively straightforward, although time-consuming. However, they are not burdened with the problems typical of Doppler measurements or tracking the magnetic field elements in the magnetograms by autocorrelation methods. Furthermore, the statistical confidence of the results can be quantitatively tested.

Since the method provides a detection of low-velocity large-scale flows, as well as the study of eddy-flow properties over a wide range of latitudes, it would be worthwhile to enlarge the number of measurements in the polar regions to get more reliable data there, especially bearing in mind the $v_{\text {mer }}(B)$ and $Q(B)$ dependencies. Furthermore, it would be desirable to extend the measurements to the whole activity cycle and to check for a possible phase dependence. Finally, an enlarged data set could enable a separate analysis for the northern and southern solar hemispheres, which were in this paper treated together.

Acknowledgements. This work was performed with the support of the Alexander von Humboldt Foundation and is related to the SOHO-EIT Proposal Brajsa_206. F. Clette and J.-F. Hochedez acknowledge the support from the Belgian OSTC and from Prodex. SOHO is a project of international cooperation between ESA and NASA, and we are grateful to the EIT team for developing and operating the instrument. We would like to thank M. Stix for helpful discussions, and to the referee (D. Mullan) whose suggestions and comments led to a significant improvement of the paper.

\section{References}

Auchère, F., Boulade, S., Koutchmy, S., et al. 1998, A\&A, 336, L57 Auchère, F., DeForest, C. E., \& Artzner, G. 2000, ApJ, 529, L115 Balthasar, H., Vázquez, M., \& Wöhl, H. 1986, A\&A, 155, 87
Beck, J. G., Gizon, L., Duvall, \& T. L., Jr. 2002, ApJ, 575, L47

Brajša, R., \& Wöhl, H. 2000, Hvar Obs. Bull., 24, 125

Brajša, R., Wöhl, H., Kasabašić, M., et al. 2000, Hvar Obs. Bull., 24, 153

Brajša, R., Wöhl, H., Vršnak, B., et al. 2001a, A\&A, 374, 309

Brajša, R., Wöhl, H., Schuck, T. J., et al. 2001b, Hvar Obs. Bull., 25, 13

Brajša, R., Wöhl, H., Vršnak, B., et al. 2002a, Sol. Phys., 206, 229

Brajša, R., Wöhl, H., Vršnak, B., et al. 2002b, A\&A, 392, 329

Brajša, R., Wöhl, H., Vršnak, B., et al. 2003, Hvar Obs. Bull., 27, in press

Brown, D. S., Parnell, C. E., DeLuca, E. E., Golub, L., \& McMullen, R. A. 2001, Sol. Phys., 201, 305

Canuto, V. M., Minotti, F. O., \& Schilling, O. 1994, ApJ, 425, 303

Delaboudinière, J.-P., Artzner, G. E., Brunaud, J., et al. 1995, Sol. Phys. 162, 291

D’Silva, S., \& Howard, R. F. 1995, Sol. Phys., 159, 63

Gilman, P. A., \& Howard, R. 1984, Sol. Phys., 93, 171

Haber, D. A., Hindman, B. W., Toomre, J., Bogart, R. S., \& Hill, F. 2001, IAU Symp., 203, 211

Haber, D. A., Hindman, B. W., Toomre, J., et al. 2002, ApJ, 570, 855

Hanslmeier, A., \& Lustig, G. 1986, A\&A, 154, 227

Howard, R. 1984, ARA\&A, 22, 131

Howard, R. F. 1991a, Sol. Phys., 135, 43

Howard, R. F. 1991b, Sol. Phys., 135, 327

Howard, R., \& Gilman, P. A. 1986, ApJ, 307, 389

Howard, R., \& LaBonte, B. J. 1980, ApJ, 239, L33

Howard, R., \& LaBonte, B. J. 1983, IAU Symp., 102, 101

Komm, R. W., Howard, R. F., \& Harvey, J. W. 1993a, Sol. Phys., 143, 19

Komm, R. W., Howard, R. F., \& Harvey, J. W. 1993b, Sol. Phys., 147, 207

LaBonte, B. J., \& Howard, R. 1982, Sol. Phys., 75, 161

Latushko, S. 1994, Sol. Phys., 149, 231

Lustig, G., \& Hanslmeier, A. 1987, A\&A, 172, 332

Lustig, G., \& Wöhl, H. 1990, A\&A, 229, 224

Lustig, G., \& Wöhl, H. 1991, A\&A, 249, 528

Lustig, G., \& Wöhl, H. 1994, Sol. Phys., 152, 221

Meunier, N., Nesme-Ribes, E., \& Collin, B. 1997, A\&A, 319, 683

Pulkkinen, P., \& Tuominen, I. 1998, A\&A, 332, 755

Rüdiger, G. 1989, Differential Rotation and Stellar Convection (Berlin: Akademie-Verlag)

Schröter, E. H. 1985, Sol. Phys., 100, 141

Snodgrass, H. B. 1991, ApJ, 383, L85

Snodgrass, H. B., \& Dailey, S. B. 1996, Sol. Phys., 163, 21

Snodgrass, H. B., \& Smith, A. A. 2000, Sol. Phys. 191, 21

Stix, M. 2002, The Sun (Berlin: Springer-Verlag)

Tuominen, J., Tuominen, I., \& Kyröläinen, J. 1983, MNRS, 205, 691

Ward, F. 1965, ApJ, 141, 534

Wöhl, H. 2002, Astron. Nachr., 323, 329

Wöhl, H., \& Brajša, R. 2001, Sol. Phys. 198, 57

Wöhl, H., Brajša, R., Vršnak, B., et al. 2001, Hvar Obs. Bull., 25, 27

Zhang, J., Kundu, M. R., \& White, S. M. 2001, Sol. Phys. 198, 347 